\title{
3D plasmonic chiral colloids $\uparrow$
}

\author{
Cite this: Nanoscale, 2014, 6, 2077

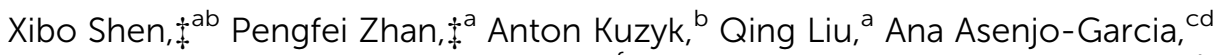 \\ Hui Zhang, ${ }^{\text {e }}$ F. Javier García de Abajo, ${ }^{\text {cf }}$ Alexander Govorov, ${ }^{\text {e Baoquan Ding }}{ }^{\star a}$ \\ and $\mathrm{Na} \mathrm{Liu}{ }^{* \mathrm{~b}}$ \\ Received 12th November 2013
}

Accepted 21st November 2013

DOI: $10.1039 / c 3 n r 06006 c$

www.rsc.org/nanoscale

3D plasmonic chiral colloids are synthesized through deterministically grouping of two gold nanorod AuNRs on DNA origami. These nanorod crosses exhibit strong circular dichroism (CD) at optical frequencies which can be engineered through position tuning of the rods on the origami. Our experimental results agree qualitatively well with theoretical predictions.

The asset of DNA as nature's designer toolkit for structural technology has been explored for several decades. ${ }^{1-5}$ The excellent control over topology and superior accuracy in templated synthesis bestow DNA the most successful molecule for programmable assembly of matter on the nanoscale. ${ }^{6-10}$ The DNA origami technique, introduced by Rothemund, ${ }^{11}$ relies on the nanoscale folding of a single-stranded viral DNA by numerous helper strands to create arbitrary 2D or 3D shapes. ${ }^{11-18}$ Due to the fact that each individual helper strand can be modified and extended to produce a sequence-dependent surface tag, DNA origami can serve as a template to assemble functionalized nanoparticles. ${ }^{19-24}$ Significant progress in DNA origami technology has been concurrent with the prompt spanning of the scope of utility and diversity of possible conjugate materials. Among them, metal nanoparticles are one of the most exciting materials, whose versatile contributions in multiple disciplines have been indisputably witnessed. ${ }^{25} \mathrm{~A}$ metal nanoparticle supports localized surface plasmons, which

\footnotetext{
${ }^{a}$ National Center for Nanoscience and Technology, No. 11 BeiYiTiao, ZhongGuanCun, Beijing 100190, China. E-mail: dingbq@nanoctr.cn

${ }^{b}$ Max-Planck-Institute for Intelligent Systems, Heisenbergstr. 3, D-70569 Stuttgart, Germany.E-mail: laura.liu@is.mpg.de

'ICFO-Institut de Ciencies Fotoniques, Mediterranean Technology Park, E-08860 Castelldefels, Barcelona, Spain

${ }^{d}$ IQFR-CSIC, Serrano 119, 28006 Madrid, Spain

'Department of Physics and Astronomy, Ohio University, Athens, Ohio 45701, USA

${ }^{f}$ ICREA - Institució Catalana de Recerca i Estudis Avançats, Barcelona, Spain

$\dagger$ Electronic supplementary information (ESI) available. See DOI: 10.1039/c3nr06006c

\$ These authors contributed equally to this work.
}

are associated with the collective oscillation of the conductive electrons in the nanoparticle. The localized nature of particle plasmons enables a vast range of useful applications, including surface-enhanced optical spectroscopies and subwavelength photonic devices, as well as medical diagnostics and therapeutics.

Recently, considerable effort has been directed towards DNA origami templated assembly of gold nanoparticles into assortments of functional nanostructures. ${ }^{26,27}$ Optical chirality in the visible spectral range is one of the most important pursuits in that it generally does not occur in natural chiral molecules. Recent seminal work was carried out by organizing gold spherical nanoparticles in a helical staircase on a DNA origami bundle. ${ }^{28}$ However, due to the weak interaction between tiny gold nanoparticles, $\mathrm{CD}$ of these plasmonic helices was rather small. Alternatively, AuNRs have been considered owing to their excellent optical properties and stronger oscillator strength. For example, AuNRs were assembled on DNA origami to form chiral nanostructures..$^{29}$ However, the achieved CD response was still quite weak and the induced chiral effect due to the presence of DNA was not considered.

Here we demonstrate 3D plasmonic chiral colloids assembled by DNA origami. Assemblies of crossed AuNRs templated by DNA origami are dispersed in a water-based solution. In each structure, two AuNRs are assembled on the opposite surfaces of a planar DNA origami sheet, forming a $90^{\circ}$ twisting angle (see Fig. 1). Such crossed AuNRs constitute the simplest chiral object in that two is the smallest number of rods that allows for 3D chirality. The plasmonic chiral colloids exhibit strong CD response at optical frequencies. In addition, the salient addressability of DNA origami allows us to engineer the structural handedness through precise control over the positions of the two AuNRs in the individual structure. ${ }^{29,30}$ This is implemented by the parallel shifting of one AuNR from one to the other end of the second AuNR with spatial accuracy on the nanoscale.

Inherent from the native assembly, the double stranded DNA (dsDNA) helices are unidirectionally oriented along the long 


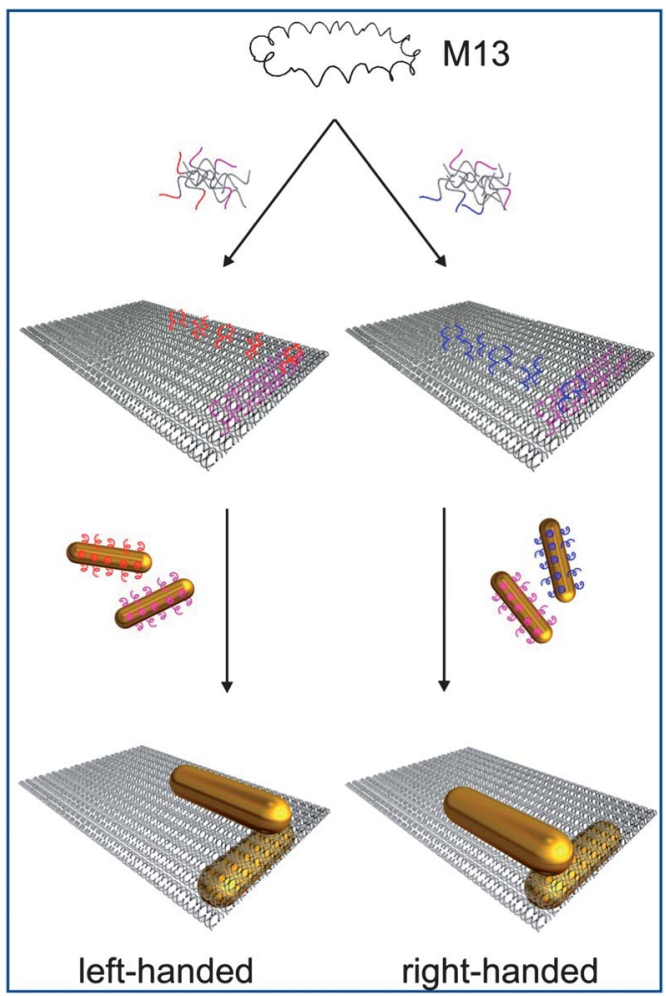

Fig. 1 Schematic illustration of the experiment. A long single DNA strand (M13) hybridizes with helper and capture strands to form a rectangular DNA origami template with well-defined binding sites. The capture strands are extended from the top and bottom surfaces of the origami template. Multiple binding sites are used to robustly assemble one gold nanorod (AuNR). The binding sites with different DNA sequences are illustrated using red, purple, and blue colors. AuNRs (nominally $40 \mathrm{~nm} \times 12 \mathrm{~nm}$ ) functionalized with corresponding complementary DNA strands are assembled at the predesignated locations on the origami template through DNA hybridization, forming left- and right-handed structures, respectively.

side of the rectangular origami sheet. Such an ordered alignment of the DNA helices provides a unique platform to investigate the interaction between DNA molecules and plasmonic nanostructures. We demonstrate that the resulting chiral molecule-induced CD is highly orientation dependent and can be controlled through varying the orientation of a single AuNR with respect to the direction of the dsDNA on the origami. Our work lays the basis for achieving complex 3D plasmonic nanomaterials with distinct designer optical functionalities. The modularity and generality of this DNA origami technology for plasmonics is thus conclusively established.

Fig. 1 illustrates the experimental scheme. Rectangular DNA origami was prepared by annealing the mixture of the M13 scaffold strand, capture strands, and helper strands at a ratio of $1: 5: 10$ from $90{ }^{\circ} \mathrm{C}$ to room temperature for 8 hours. The product was then purified with a filter device $[100 \mathrm{kDa}$, molecular weight cutoff (MWCO), Amicon, Millipore] to remove the extra helper and capture stands. The dimensions of the rectangular origami were approximately $90 \mathrm{~nm} \times 60 \mathrm{~nm} \times 2 \mathrm{~nm}$. DNA capture strands with carefully designed sequences were extended from the two surfaces of the rectangular DNA template. For each AuNR, multiple binding sites were used in order to achieve robust assembly [see ESI†]. In Fig. 1, red, purple, and blue colors are used to highlight these three different groups of binding sites. Red and blue groups are on the top surface, whereas the purple group is on the bottom surface of the DNA origami. AuNRs (nominally $40 \mathrm{~nm} \times 12 \mathrm{~nm}$ ) modified with corresponding complementary DNA strands were assembled through DNA hybridization at the designated binding sites on the DNA origami. The absence of symmetry planes enforced by the $90^{\circ}$ twisting angle results in two plasmonic enantiomers: a left handed one (AuNRs placed at groups red and purple) and a right-handed one (AuNRs placed at groups blue and purple), respectively. DNA origami structures dressed with single AuNRs were obtained by placing one AuNR at the red, purple, or blue group, following similar experimental procedures. The purified DNA origami and functionalized AuNRs were then mixed and annealed from $43{ }^{\circ} \mathrm{C}$ to $23{ }^{\circ} \mathrm{C}$ for 30 cycles over 48 hours. ${ }^{30}$ After DNA hybridization, the AuNRs were immobilized on the DNA origami template at the desired positions (see ESI†̈).

Subsequently, the annealed products were analyzed and separated by agarose gel electrophoresis. The gel image is shown in Fig. 2a. Lanes 1 and 2 correspond to the M13 strand and the rectangular DNA origami, respectively. Lane 3, 4, and 5 correspond to the DNA origami structures dressed with single AuNRs at the red, purple, and blue groups. Lanes 6 and 7

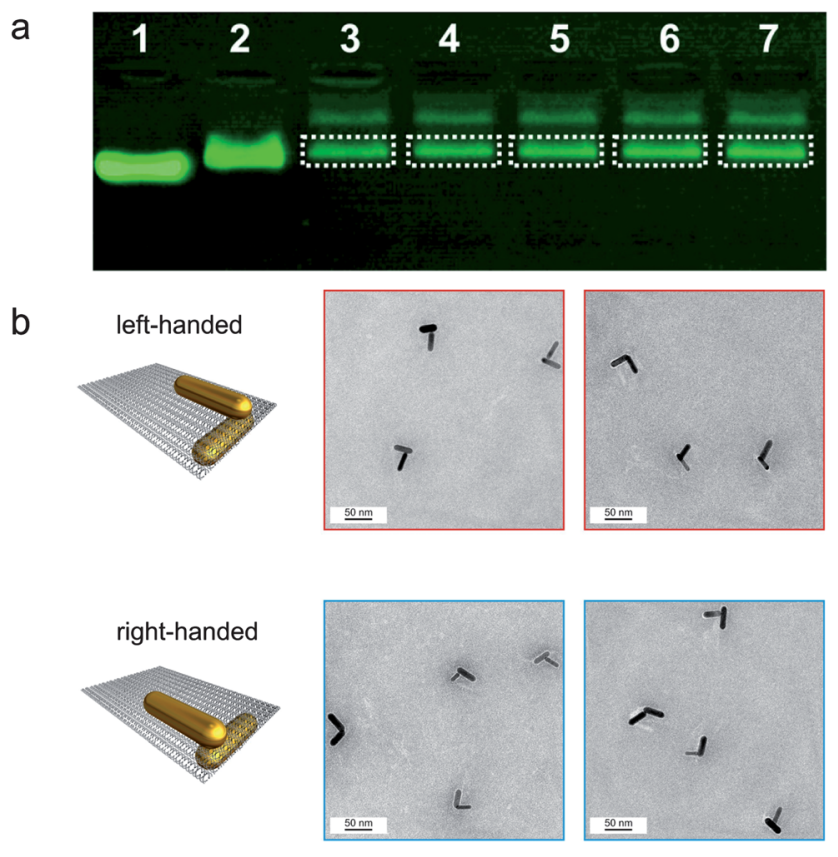

Fig. 2 Agarose gel image and TEM images of the left- and righthanded AuNR structures. (a) Ethidium bromide-stained agarose gel of the products. Lane 1: M13. Lane 2: rectangular DNA origami. Lanes 3, 4, and 5: single AuNR dressed DNA origami at binding groups of red, purple, and blue (see Fig. 1). Lane 6: left-handed crossed AuNR structures. Lane 7: right-handed crossed AuNR structures. The target products are contained in the bands highlighted by the white dashed lines. (b) Up: TEM images of the left-handed crossed AuNRs. Down: TEM images of the right-handed crossed AuNRs. 
contain the left- and right-handed crossed rods, respectively. The target gel bands highlighted by white-dashed lines in Fig. 2a were then cut and extracted by electro-elution with dialysis tubing membranes (MWCO: 50K). Fig. 2b shows the transmission electron microscopy (TEM) images of the left- and right-handed crossed rod structures. The TEM images match our designs well. It is noteworthy that in some structures the twisting angle between the two AuNRs is not exactly $90^{\circ}$, stemming from the DNA origami deformation by the AuNRs during the drying process on the TEM grids. Nevertheless, as revealed by the TEM images, the position and orientation accuracy of the AuNRs on the DNA origami is very good.

CD measurements were carried out with a quartz cuvette (0.5 cm path length) using a J-810 Circular Dichroism Spectrometer. All the samples were dispersed in $1 \times \mathrm{TAE} / \mathrm{Mg}^{2+}$ buffer (water-based). The extinction spectra of the samples can be found in the ESI. $\dagger$ Fig. 3a shows the CD spectra of the lefthanded (red) and right-handed (blue) crossed rods. It is evident that the two plasmonic enantiomers display bisignated spectral bands with opposite chirality. More specifically, the left-handed sample shows a larger CD response compared to the righthanded one.

In fact, the configuration of our crossed AuNRs is strongly reminiscent of the interacting chromophores in the quantum exciton-coupling theory used by Kuhn and Born to interpret optical activity of natural chiral molecules. In order to gain deeper insight into the CD mechanism, a classical electrodynamics theory calculation was carried out to describe the plasmonic CD generated in our plasmonic assemblies. ${ }^{\mathbf{2 8 , 3 1 , 3 2}} \mathrm{We}$ utilized the Discrete Dipole Approximation (DDA) method $^{33-36}$ to obtain fully numerical results. In the aqueous solution, the plasmonic assemblies are randomly oriented and illuminated by light of fixed direction. This is equivalent to averaging over the results of a single assembly interacting with light for all possible incident directions. The details of the computational process can be found in a recent paper. ${ }^{32}$

The longitudinal resonance of the AuNRs is located around $730 \mathrm{~nm}$ as shown in Fig. 3a. When interacting with circularly polarized light, the near-field coupling between the two AuNRs results in symmetric and antisymmetric hybrid modes (see Fig. 3c). The symmetric mode is blue-shifted compared to the resonance of the individual AuNRs. This is caused by Coulomb repulsion of the charges, which oscillate in phase in the two AuNRs. On the other hand, the antisymmetric mode is redshifted due to anti-phase oscillations of the charges in the two AuNRs. Such a mode splitting gives rise to a characteristic bisignated CD couplet, centered at the extinction wavelength of the AuNRs around $730 \mathrm{~nm} \cdot{ }^{37,38}$ Another CD signature is visible at a shorter wavelength around $520 \mathrm{~nm}$ (see Fig. 3a), which is due to the plasmonic excitations along the transverse direction of the AuNRs. The CD response at this wavelength is substantially smaller, as expected from the weaker strength of the transverse mode compared to that of the longitudinal mode (see the inset of Fig. 3a). Fig. 3b presents the calculated results. The calculations qualitatively agree with the experiment. The line widths of the experimental CD spectra are broader than those of the calculated CD spectra mainly due to the inhomogeneous size a

b
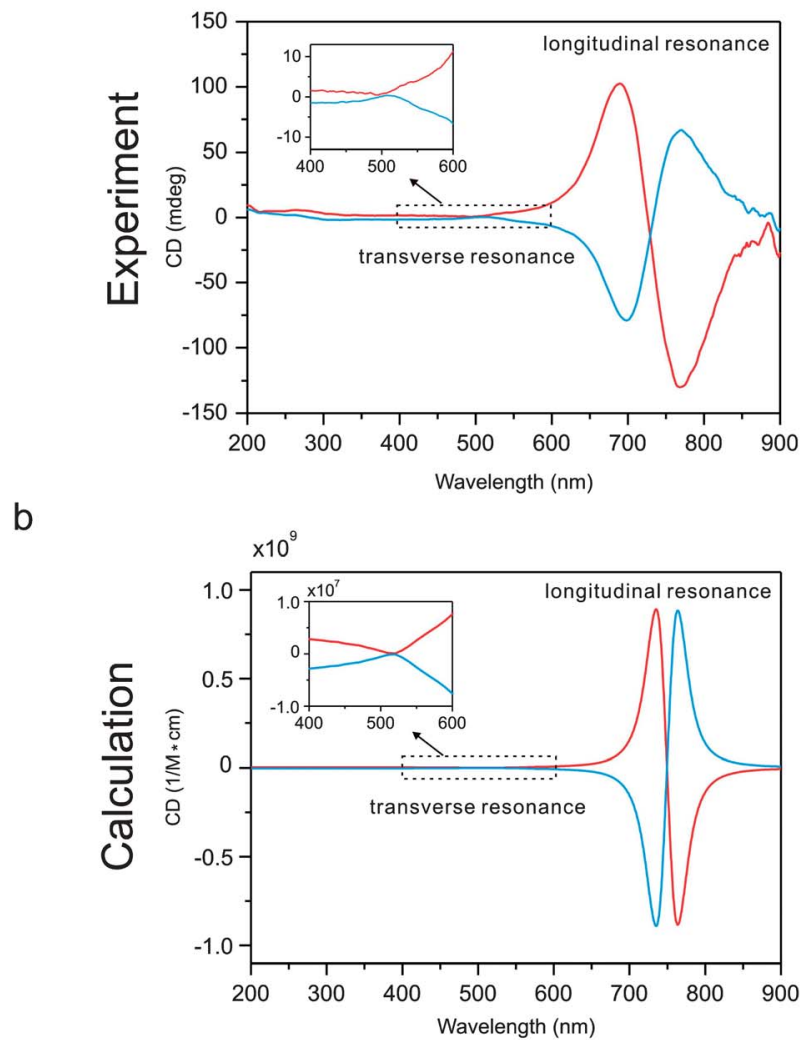

C

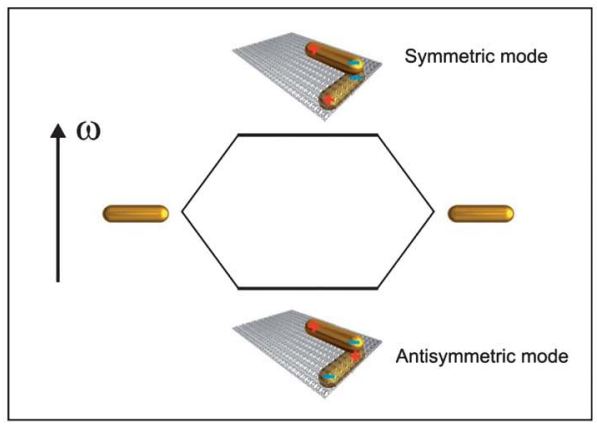

Fig. 3 Optical characterization of the 3D plasmonic chiral structures. (a) Measured CD spectra of the left- (red) and right-handed (blue) crossed AuNRs. Strong CD is observed around $730 \mathrm{~nm}$, which corresponds to the longitudinal resonance of the AuNRs. The CD response of the left-handed sample is stronger than that of the right-handed one. Smaller CD is observed around $520 \mathrm{~nm}$, which corresponds to the transverse resonance of the AuNRs. (b) Calculated CD spectra of the left- (red) and right-handed (blue) crossed AuNRs. (c) Hybridization of the plasmons in the crossed AuNRs forms symmetric and antisymmetric plasmonic modes.

distribution of the synthesized AuNRs. Also, the CD spectra are mirrored for the left- and right-handed structures in the calculations. At this stage, it is still challenging to model the DNA origami sheet in the theoretical calculations, which might be a main reason for the observed larger CD response in the lefthanded sample compared to that in the right-handed one in the 
experiment. Still, CD resulting from the 3D plasmonic chiral nanostructures plays a dominating role with respect to any possible induced $\mathrm{CD}$, given the markedly large CD amplitudes in the experiment. Another possible reason could be due to the preferred folding dynamics of the DNA origami in the aqueous solution, ${ }^{39-41}$ which likely renders the left-handed configuration more favorable. Further research on this front is still needed.

It has been theoretically proposed that the optical activity of natural chiral molecules can be both amplified and transferred to the resonance position of a plasmonic particle via their Coulomb interaction. Such an interaction is highly anisotropic and dependent on the orientation of the chiral molecule with respect to the plasmonic particle. ${ }^{\mathbf{4 2 , 4 3}}$ For a long time, rigorous experimental verification of this orientation aspect has remained elusive, due to the significant challenge in controlling molecular orientations on the nanoscale. Fortunately, DNA origami offers a native platform to orient DNA strands through directed self-assembly. In our case, after DNA hybridization the dsDNA is unidirectionally aligned along the long side of the rectangular origami sheet. Such an orientationally engineerable chiral system constitutes a fundamental basis for experimental investigation of chiral molecule-induced CD. The strong CD in Fig. 3a that mainly arises from the $3 \mathrm{D}$ chiral geometry of the crossed AuNRs overshadows the characterization of the chiral molecule-induced CD, which possesses substantially smaller response. We therefore utilize a simpler system, which contains an achiral plasmonic element, a single AuNR on DNA origami, to study this aspect.

Fig. 4 shows the experimental CD results of the DNA origami dressed with single AuNRs. A AuNR is oriented either parallel or perpendicular to the long side of the DNA origami (i.e., the axis of the dsDNA helices). It is apparent in Fig. 4 that the two orthogonal configurations result in bisignated CD spectra centered at around $730 \mathrm{~nm}$, which are flipped when switching

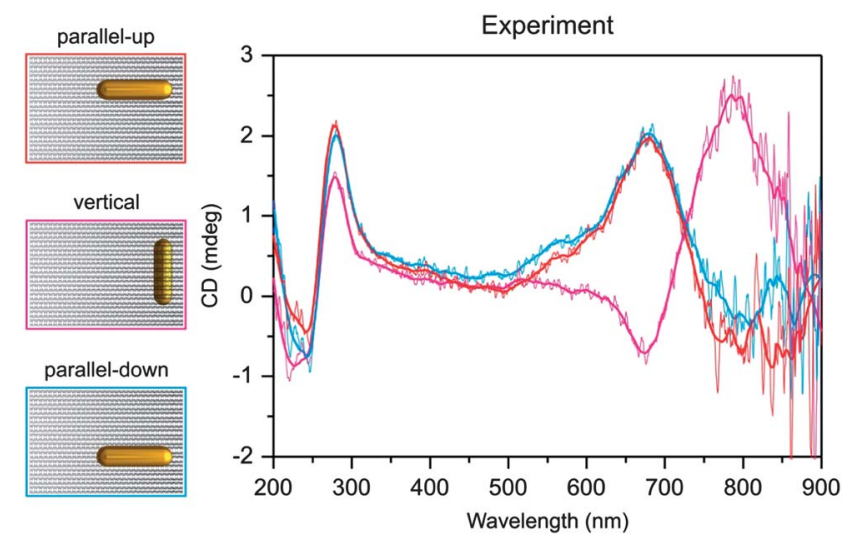

Fig. 4 Optical characterization of the DNA origami dressed with single AuNRs. Chiral molecule-induced CD is clearly observable centered at around $730 \mathrm{~nm}$. CD in the parallel AuNR cases (red and blue) shows opposite chirality compared to that in the vertical AuNR case (purple). The experimental results clearly demonstrate that the chiral moleculeinduced CD is highly orientation dependent. The smoothed lines that are imposed on the raw data were obtained using the Savitzky-Golay method. between the parallel and perpendicular orientations. More specifically, when the AuNR is assembled parallel to the dsDNA helices, it generates a peak-to-dip line shape, giving rise to lefthanded induced CD. In contrast, when the AuNR is assembled perpendicular to the dsDNA helices, the CD spectrum is characterized by a dip-to-peak line shape, revealing a right-handed induced CD. As a result, the anisotropic nature of the AuNRs enables a clear identification of the orientation dependence of the chiral molecule-induced CD.

\section{Conclusions}

The interdisciplinary character of structural DNA technology has prompted it to the forefront of nanoscience and nanotechnology. The programmability of DNA offers unprecedented spatial control over discrete constituents down to the nanoscale. Simultaneously, this capacity also provides an excellent playground to explore exotic optical functionalities of artificial nanostructures, in particular, chirality at optical frequencies. The ubiquitous relationship between the 3D configurations of plasmonic chiral objects and their chirality will be the driving force to develop a new generation of 3D plasmon rulers, which hold great potential for structural analysis, pharmacology, and biomachinery.

\section{Acknowledgements}

B. D. was supported by National Basic Research Program of China (973 Program, 2012CB934000), 100-Talent Program of Chinese Academy of Sciences, National Science Foundation China (21173059, 91127021) and Beijing Natural Science Foundation (2122057). N. L. and X. B. S. were supported by the Sofja Kovalevskaja Award from the Alexander von HumboldtFoundation. N. L. and A. K. were supported by the Marie Curie CIG Fellowship. F. J. G. A. and A. A. G. acknowledge support from the Spanish MEC (MAT2010-14885 and Consolider NanoLight.es). A. A. G. acknowledges financial support through FPU from the Spanish ME. H. Z. and A. O. G. were supported by the U. S. Army Research Office under the grant number W911NF-12-1-0407 and by Volkswagen Foundation (Germany). Use of the Center for Nanoscale Materials by A. O. G and H. Z. was supported by the U. S. Department of Energy, Office of Science, Office of Basic Energy Sciences, under Contract No. DEAC02-06CH11357.

\section{Notes and references}

1 N. C. Seeman, Nature, 2003, 421, 427-431.

2 N. C. Seeman, Nano Lett., 2010, 10, 1971-1978.

3 A. V. Pinheiro, D. Han, W. M. Shih and H. Yan, Nat. Nanotechnol., 2011, 6, 763-772.

4 A. Condon, Nat. Rev. Genet., 2006, 7, 565-575.

5 Y. G. Ke, L. L. Ong, W. M. Shih and P. Yin, Science, 2012, 338, 1177-1183.

6 J. J. Storhoff and C. A. Mirkin, Chem. Rev., 1999, 99, 18491862.

7 N. C. Seeman, Annu. Rev. Biochem., 2010, 79, 65-87. 
8 K. V. Gothelf and T. H. LaBean, Org. Biomol. Chem., 2005, 3, 4023-4037.

9 Y. H. Roh, R. C. H. Ruiz, S. M. Peng, J. B. Lee and D. Luo, Chem. Soc. Rev., 2011, 40, 5730-5744.

10 B. Sacca and C. M. Niemeyer, Chem. Soc. Rev., 2011, 40, 59105921.

11 P. W. Rothemund, Nature, 2006, 440, 297-302.

12 S. M. Douglas, H. Dietz, T. Liedl, B. Hogberg, F. Graf and W. M. Shih, Nature, 2009, 459, 414-418.

13 H. Dietz, S. M. Douglas and W. M. Shih, Science, 2009, 325, 725-730.

14 T. Torring, N. V. Voigt, J. Nangreave, H. Yan and K. V. Gothelf, Chem. Soc. Rev., 2011, 40, 5636-5646.

15 D. R. Han, S. Pal, J. Nangreave, Z. T. Deng, Y. Liu and H. Yan, Science, 2011, 332, 342-346.

16 D. R. Han, S. Pal, Y. Yang, S. X. Jiang, J. Nangreave, Y. Liu and H. Yan, Science, 2013, 339, 1412-1415.

17 B. Sacca and C. M. Niemeyer, Angew. Chem., Int. Ed., 2012, 51, 58-66.

18 F. C. Simmel, Front Life Sci, 2012, 6, 3-9.

19 B. Q. Ding, Z. T. Deng, H. Yan, S. Cabrini, R. N. Zuckermann and J. Bokor, J. Am. Chem. Soc., 2010, 132, 3248-3249.

20 S. Pal, Z. T. Deng, B. Q. Ding, H. Yan and Y. Liu, Angew. Chem., Int. Ed., 2010, 49, 2700-2704.

21 H. Bui, C. Onodera, C. Kidwell, Y. Tan, E. Graugnard, W. Kuang, J. Lee, W. B. Knowlton, B. Yurke and W. L. Hughes, Nano Lett., 2010, 10, 3367-3372.

22 S. H. Ko, K. Du and J. A. Liddle, Angew. Chem., Int. Ed., 2013, 52, 1193-1197.

23 N. Stephanopoulos, M. H. Liu, G. J. Tong, Z. Li, Y. Liu, H. Yan and M. B. Francis, Nano Lett., 2010, 10, 2714-2720.

24 Z. Deng, A. Samanta, J. Nangreave, H. Yan and Y. Liu, J. Am. Chem. Soc., 2012, 134, 17424-17427.

25 S. J. Tan, M. J. Campolongo, D. Luo and W. L. Cheng, Nat. Nanotechnol., 2011, 6, 268-276.

26 X. B. Shen, C. Song, J. Y. Wang, D. W. Shi, Z. A. Wang, N. Liu and B. Q. Ding, J. Am. Chem. Soc., 2012, 134, 146149.
27 X. B. Shen, A. Asenjo-Garcia, Q. Liu, Q. Jiang, F. J. G. de Abajo, N. Liu and B. Q. Ding, Nano Lett., 2013, 13, 2128-2133.

28 A. Kuzyk, R. Schreiber, Z. Y. Fan, G. Pardatscher, E. M. Roller, A. Hogele, F. C. Simmel, A. O. Govorov and T. Liedl, Nature, 2012, 483, 311-314.

29 X. Lan, Z. Chen, G. Dai, X. Lu, W. Ni and Q. Wang, J. Am. Chem. Soc., 2013, 135, 11441-11444.

30 S. Pal, Z. T. Deng, H. N. Wang, S. L. Zou, Y. Liu and H. Yan, J. Am. Chem. Soc., 2011, 133, 17606-17609.

31 Z. Fan and A. O. Govorov, Nano Lett., 2010, 10, 2580-2587.

32 Z. Fan, H. Zhang and A. O. Govorov, J. Phys. Chem. C, 2013, 117, 14770-14777.

33 P. J. Flatau and B. T. Draine, Opt. Express, 2012, 20, 12471252.

34 B. T. Draine and P. J. Flatau, J. Opt. Soc. Am. A, 1994, 11, 1491-1499.

35 The DDA code was taken from the open source at http:// www.astro.princeton.edu/ draine/DDSCAT.html, accessed July 2013.

36 B. T. Draine and P. J. Flatau, User Guide for the Discrete Dipole Approximation Code DDSCAT 7.2, http://arXiv.org/abs/ 1202.3424, accessed July 2013.

37 A. Guerrero-Martinez, J. L. Alonso-Gomez, B. Auguie, M. M. Cid and L. M. Liz-Marzan, Nano Today, 2011, 6, 381400.

38 B. Auguie, J. L. Alonso-Gomez, A. Guerrero-Martinez and L. M. Liz-Marzan, J. Phys. Chem. Lett., 2011, 2, 846-851.

39 C. E. Castro, F. Kilchherr, D. N. Kim, E. L. Shiao, T. Wauer, P. Wortmann, M. Bathe and H. Dietz, Nat. Methods, 2011, 8, 221-229.

40 D. N. Kim, F. Kilchherr, H. Dietz and M. Bathe, Nucleic Acids Res., 2012, 40, 2862-2868.

41 Z. Li, L. Wang, H. Yan and Y. Liu, Langmuir, 2012, 28, 19591965.

42 A. O. Govorov, Z. Y. Fan, P. Hernandez, J. M. Slocik and R. R. Naik, Nano Lett., 2010, 10, 1374-1382.

43 A. O. Govorov and Z. Fan, ChemPhysChem, 2012, 13, 25512560. 